\title{
Descrição preliminar do cenário da comunicação científica na América Latina e Caribe
}

\author{
Preliminary description of scientific communication scenario in Latin \\ America and Caribbean
Descripción preliminar del escenario de la comunicación científica en América Latina y Caribe

\author{
Michelli Costa | michellicosta11@gmail.com \\ Universidade de Brasília (UnB), Faculdade de Ciência da Informação, Programa de Pós-Graduação em Ciência da \\ Informação. Brasília, DF, Brasil \\ Fernando César Lima Leite | fernandodfc@gmail.com \\ Universidade de Brasília (UnB), Faculdade de Ciência da Informação, Programa de Pós-Graduação em Ciência da \\ Informação. Brasília, DF, Brasil
}

\section{RESUMO}

O modelo de colonização instaurado na América Latina e no Caribe influenciou profundamente o desenvolvimento da ciência e tecnologia (C\&T) bem como dos processos constituídos para fazer fluir a informação que é necessária e que resulta da produção do conhecimento científico. Ao serem considerados como a atividade capaz de promover fluxos de informação imprescindíveis à capacidade de fazer avançar a C\&T, os processos de comunicação científica representam indicadores do desenvolvimento científico de uma área do conhecimento ou região. Partindo desse pressuposto, este artigo tem por objetivo relatar e discutir resultados de levantamento sobre o cenário da comunicação científica na América Latina e no Caribe. Para tanto, foram coletados e analisados dados sobre investimento/financiamento em C\&T, uma série de artigos de periódicos, títulos de periódicos e patentes na base de dados da Red de Indicadores de Ciencia y Tecnología - Iberoamericana e Interamericana (Ricyt), no Directory of Open Access Journals (DOAJ) e em páginas web das agências de fomento da região. Entre os resultados é possível indicar que Brasil, Argentina e México concentram maior parte do investimento em C\&T; o crescimento das publicações latino-americanas se deve, em grande parte, ao crescimento das publicações científicas no Brasil; a maior parte da atividade científica e tecnológica da América Latina é financiada com recursos públicos; a região tem apresentado consideráveis avanços nos últimos dez anos, tanto em investimento em pesquisa como no desenvolvimento de mecanismos para a comunicação científica.

Palavras-chave: indicadores de ciência e tecnologia; comunicação científica; literatura científica; América Latina e Caribe. 


\section{ABSTRACT}

The colonization model implemented in Latin America influenced the development of science and technology as well as the processes related to the communication of information that is required and that results from the production of scientific knowledge. Is so far as the scientific communication processes are considered an activity that promote essential information flows to advance of science and technology, they represent indicators of scientific development of an area of knowledge or region. Considering this assumption, this article aims to report and discuss the scenario of scientific communication in Latin America and Caribbean. For this purpose, we collected and analyzed data on investment/financing in science and technology, a set of journal articles, scientific journals and patents in the database of the Red de Indicadores de Ciencia y Tecnología - Iberoamericana e Interamericana (Ricyt), in the Directory of Open Access Journals (DOAJ) and in web pages of development agencies in the region. The results indicate that Brazil, Argentina and Mexico concentrate most of the investment in science and technology; the growth of Latin American publications is due in large part to the growth of scientific publications in Brazil; most of the scientific and technological activities in Latin America is financed by public funds; the region has shown considerable progress in the last ten years, both in research investment and in developing of mechanisms for scientific communication. Keywords: indicators of science and technology; scientific communication; literature; Latin America and Caribbean.

\section{RESUMEN}

El modelo de colonización implementada en América Latina y Caribe influyó profundamente en el desarrollo de la ciencia y la tecnología y también los procesos para hacer circular la información que se requiere y que resulta de la producción de conocimiento científico. Considerados como la actividad capaz de promover flujos de información esenciales para la capacidad de avanzar la ciencia y la tecnología, los procesos de comunicación de la ciencia representan indicadores de desarrollo científico de una área de conocimiento o de una región. Basado en este presupuesto, este artículo tiene como objetivo informar y analizar los resultados de una investigación sobre el escenario de la comunicación científica en América Latina y Caribe. Para ello, recogemos y analizamos datos acerca de inversión/financiación en la ciencia y la tecnología, una serie de artículos de revistas, publicaciones científicas y patentes en la base de datos de la Red de Indicadores de Ciencia y Tecnología - Iberoamericana e Interamericana (Ricyt), en el Directory of Open Access Journals (DOAJ) y en páginas web de los organismos de fomento de la región. Entre los resultados es posible indicar que Brasil, Argentina y México concentran la mayor parte de la inversión en la ciencia y la tecnología; el crecimiento de las publicaciones de América Latina se debe en gran parte al crecimiento de las publicaciones científicas en Brasil; la mayor parte de la actividad científica y tecnológica en América Latina es financiada por fondos públicos; la región ha mostrado un progreso considerable en los últimos diez años, tanto en la inversión en investigación como en el desarrollo de mecanismos para la comunicación científica. Palabras clave: indicadores de ciencia y tecnología; Comunicación científica; Literatura; América Latina y Caribe

Contribuição dos autores:

- Concepção e desenho do estudo: o artigo é resultado de pesquisa de mestrado de autoria da autora Michelli Costa sob orientação do coautor Fernando Leite. O estudo concebido pela dupla, inclusive seu planejamento.

- $\quad$ Aquisição, análise ou interpretação dos dados: os dados foram adquiridos, analisados e interpretados pela autora Michelli Costa sob constante acompanhamento e revisão do orientador.

- Redação do manuscrito: o manuscrito é parte constituindo da dissertação de mestrado da autora Michelli Costa, também responsável primeira pela redação do manuscrito. A versão inicial foi submetida ao orientador, que procedeu não apenas sua revisão teórica mas também interviu incorporando trechos e retirando outros.

- Revisão crítica do conteúdo intelectual: o segundo autor, como orientador, procedeu revisão macro e teórica do manuscrito e, somente então, foi realizada submissão à revista.

Declaração de conflito de interesses: Não há conflitos de interesses a serem declarados.

Fontes de financiamento: A pesquisa apresentada não foi objeto de financiamento.

Considerações éticas: Não há considerações éticas a serem declaradas.

Agradecimento/Contribuições adicionais: Não se aplica.

Histórico do artigo: Submetido: 27.jun.2016 | Aceito: 06.fev.2017 | Publicado: 30.jun.2017

Apresentação anterior: Não se aplica.

Licença CC BY-NC atribuição não comercial. Com essa licença é permitido acessar, baixar (download), copiar, imprimir, compartilhar, reutilizar e distribuir os artigos, desde que para uso não comercial e com a citação da fonte, conferindo os devidos créditos de autoria e menção à Reciis. Nesses casos, nenhuma permissão é necessária por parte dos autores ou dos editores. 


\section{Introdução}

A comunicação científica é entendida como parte fundamental da própria ciência. De acordo com Roosendaal e Geurts ${ }^{1}$, sua importância relaciona-se com suas próprias funções básicas. A primeira delas é o registro da autoria, momento em que se estabelece a propriedade intelectual. A segunda função diz respeito à certificação, momento em que o conhecimento gerado é submetido ao escrutínio de outros pesquisadores de modo que seja garantia da qualidade e validação dos conhecimentos gerados (avaliação por pares). A terceira função básica, por sua vez, é a circulação, em que são promovidas as condições de acesso aos resultados de pesquisa. Por fim, a quarta função é o arquivamento, que promove a preservação do conhecimento gerado para que possa ser utilizado e reutilizado em novos ciclos de produção do conhecimento no futuro. Dessa maneira, a comunicação científica pode ser entendida como ação cíclica indissociável dos processos de produção da ciência, presente nos diferentes estágios das práticas de pesquisa.

De acordo com Garvey², para que uma nova ideia seja consideravelmente relevante na ciência, é necessário que ela seja comunicada. Esta comunicação precisa ser compreendida e verificada por outros cientistas para que possa ser considerada como válida e ser usada para novas pesquisas, que, por sua vez, precisarão igualmente ter seus resultados comunicados. Para o autor, os processos que envolvem a comunicação científica iniciam-se com o uso de um conhecimento já registrado, de forma a possibilitar a produção de um novo conhecimento, que precisa ser disseminado para que possa ser utilizado por outros pesquisadores, concluindo assim o seu ciclo.

De acordo com Chan e Costa $^{3}$, o acesso à ciência e tecnologia (C\&T) é necessário para diminuir os problemas dos países em desenvolvimento, como é o caso dos países da América Latinai. Segundo os autores, esses países estão à margem da produção de C\&T devido ao baixo investimento em pesquisa e às dificuldades de acesso à literatura científica internacional em decorrência dos altos custos das assinaturas dos periódicos mais relevantes. Os autores apresentaram dados de 2004, publicados pela Organização Mundial da Saúde, que demonstravam que, entre 1999 a 2004, 56\% dos países com PIB inferior a mil dólares não fizeram assinatura dos principais periódicos científicos.

Ao serem considerados como a atividade capaz de promover fluxos de informação imprescindíveis à capacidade de fazer avançar a C\&T, os processos de comunicação científica representam indicadores do desenvolvimento científico de uma área do conhecimento ou região. Partindo desse pressuposto, este artigo tem por objetivo relatar e discutir resultados de levantamento sobre o cenário da comunicação científica na América Latina.

\section{Procedimentos metodológicos}

O propósito do estudo é descritivo, tal como entendem Fernandes e Gomes ${ }^{4}$. Segundo os autores, este tipo de pesquisa retrata e analisa o objeto e suas relações com os fenômenos. Nesse sentido, a pesquisa é feita para que seja conhecido qual é o fenômeno, no caso a comunicação científica, como ele acontece e com qual intensidade. Para efeitos deste trabalho, o cenário da comunicação científica foi descrito a partir do conjunto de atividades relacionadas com duas dimensões: i) investimento/financiamento em C\&T e ii) informação científica e tecnológica (artigos de periódicos, títulos de periódicos e patentes).

Os dados que permitiram descrever o cenário da comunicação científica na América Latina, a partir das duas dimensões definidas, foram coletados em páginas web das agências de fomento, na base de dados da

i De acordo com o relatório do FMI de 2012, todos os países da América Latina considerados neste estudo são países emergentes, exceto Cuba, que não foi analisada no estudo. Disponível em: http://www.imf.org/en/Publications/REO/WH/ Issues/2017/02/01/Latin-America-and-the-Caribbean2 
Red de Indicadores de Ciencia y Tecnología - Iberoamericana e Interamericana (Ricyt) e no Directory of Open Access Journals (DOAJ). Os dados coletados foram submetidos à análise estatística descritiva.

No contexto do presente trabalho, a América Latina foi delimitada em um conjunto de 24 países da América do Sul, da América Central e do Caribe. Esse conjunto foi definido a partir da intersecção dos grupos de países que compreendem a América Latina apresentados pela Organização das Nações Unidas para a Educação, a Ciência e a Cultura (Unesco) $)^{\mathrm{ii}}$ e pela Ricytiii.

Considerando que o escopo da Ricyt não é restrito à América Latina e abrange países da América do Norte mais Espanha e Portugal, foi necessário fazer a intersecção das definições das duas organizações. Para a composição do conjunto dos países que compõem a América Latina, realizou-se uma combinação entre a definição dos países da América Latina da Unesco e da Ricyt, esta última incluindo países da América Central e do Sul (Figura 1).

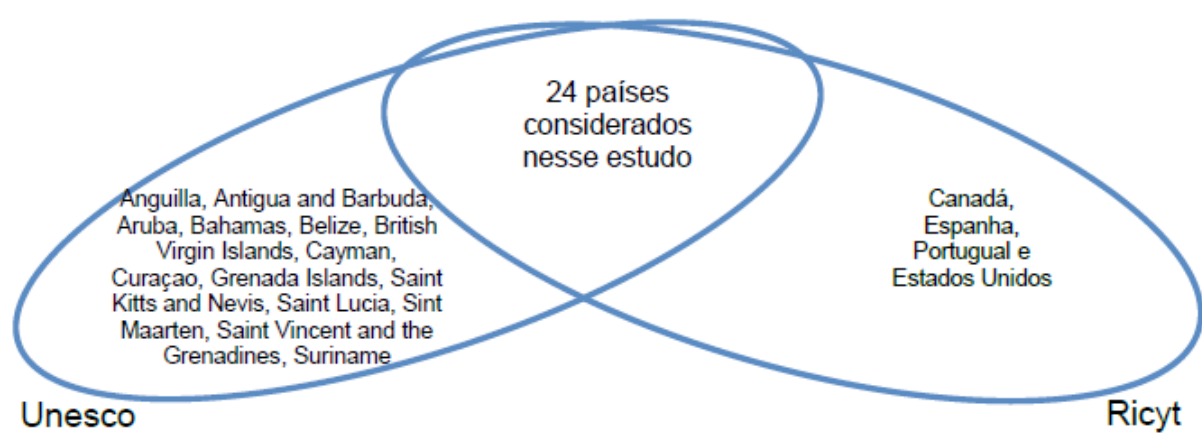

Figura 1 - Conjunto dos países da América Latina e Caribe

Fonte: Elaborada pelos autores (2017)

Sob essa perspectiva, os 24 países da América Latina que foram analisados são: Argentina, Barbados, Bolívia, Brasil, Chile, Colômbia, Costa Rica, Cuba, República Dominicana, Equador, Guatemala, Guiana, Honduras, Haiti, Jamaica, México, Nicarágua, Panamá, Peru, Paraguai, El Salvador, Trinidad y Tobago, Uruguai e Venezuela. Os países têm por característica comum o fato de terem sido colonizados por países europeus, sendo a maioria deles pela Espanha e os demais por Portugal, Inglaterra, França e Holanda. Devido a esse processo, os países latino-americanos têm como idioma oficial o espanhol, o português, o inglês, o castelhano, o francês, o guarani e o crioulo ${ }^{5}$

\section{Produção de conhecimento na América Latina}

No que diz respeito à produção de conhecimento científico, segundo a perspectiva histórica traçada por Lopes ${ }^{6}$, em decorrência da colonização europeia a América Latina teve seus sistemas originais de produção do conhecimento destruídos e foram impostas diversas barreiras para acompanhar o desenvolvimento científico e tecnológico de outras regiões. Isso porque suas atividades científicas foram interrompidas com a chegada dos portugueses e espanhóis no século XVI. Segundo o autor, até então, a região tinha conquistado importantes níveis de desenvolvimento material e cultural por alguns povos. Um exemplo foram os maias que, antes da chegada dos colonizadores, haviam produzido conhecimentos avançados nas áreas de matemática, astronomia, arquitetura, agricultura e engenharia.

\footnotetext{
ii A Unesco foi criada com o objetivo de garantir a paz por meio da cooperação intelectual entre as nações, acompanhando o desenvolvimento mundial e auxiliando os Estados-membros. Disponível em: http://www.onu.org.br/onu-no-brasil/unesco/.

iii A Ricyt é uma rede de instituições e organismos nacionais de ciência e tecnologia dos países da América e da Península Ibérica. Seu objetivo é promover o desenvolvimento e uso dos instrumentos para a medição e análise da ciência e da tecnologia na Ibero-América. Disponível em: http://www.ricyt.org/.
} 
Além da destruição dos sistemas originais de produção do conhecimento, a região passou pelo processo de industrialização tardiamente em comparação aos países do Norte do mundo. De acordo com Lopes o conhecimento científico e tecnológico necessário para a industrialização na América Latina já vinha incorporado ao maquinário e nas técnicas importadas. Esse fator não proporcionava ou estimulava o desenvolvimento de conhecimentos e capacidades para o aperfeiçoamento e manutenção das tecnologias, muito menos para o desenvolvimento de novas competências.

Esse contexto foi determinante para a configuração do desenvolvimento científico e tecnológico da região, como defendido por Lopes $^{6}$, Erber $^{7}$ e Zúñiga ${ }^{8}$. De acordo com Zúñiga ${ }^{8}$, tais aspectos históricos são característicos do "terceiro mundo" e, em especial, da América Latina. Além disso, Erber ${ }^{7}$ chama a atenção para o fato de que "a recorrência de alguns problemas ao longo de várias décadas permite caracterizálos como problemas estruturais, onde operam características de cumulatividade, rigidez e fixação de trajetórias". Portanto, para pensar a produção e comunicação da ciência na região, é necessário considerar sua totalidade histórica.

Tendo em vista as características históricas de colonização e de produção de C\&T, Erber ${ }^{7}$ faz uma comparação entre a C\&T da região e a do contexto internacional, tendo como referência os Estados Unidos da América (EUA). Segundo levantamento realizado pelo autor em 1996, os investimentos em C\&T na América Latina corresponderam a apenas $5 \%$ do total dos investimentos feitos nos EUA, o número de patentes concedidas representava $0,15 \%$ do total de patentes dos EUA e a quantidade de publicações científicas equivalia a cerca de $2 \%$ do total de publicações dos EUA. O autor conclui que existe uma desproporção entre o peso econômico da região e seus investimentos em C\&T. A partir da comparação da quantidade de patentes com a quantidade de publicações científicas, Erber $^{7}$ observou um maior desempenho em atividades científicas do que em atividades tecnológicas.

É importante mencionar que o estudo de $\operatorname{Erber}^{7}$ considerou apenas dados do Brasil, Argentina e México. Erber $^{7}$ justifica a seleção dos países com os dados da Tabela 1, na qual demonstrou que os três países "respondem por uma esmagadora parcela dos gastos em C\&T, publicações e patentes" da região. Em 1996, a soma do produto interno bruto (PIB) desses países chegava a mais de $75 \%$ do PIB de toda a região e os seus investimentos em produção de C\&T, bem como seus resultados medidos em publicações científicas e patentes, giravam em torno de $80 \%$ do total da América Latina.

Tabela 1. A concentração de C\&T da América Latina: participação de Argentina, Brasil e México como \% do total regional - 1996

\begin{tabular}{|c|c|c|c|c|c|}
\hline País & PIB & $\begin{array}{l}\text { Gastos em } \\
\text { C\&T }\end{array}$ & $\begin{array}{l}\text { Publicações } \\
\text { científicas }\end{array}$ & \multicolumn{2}{|c|}{ Patentes concedidas } \\
\hline & & & & Residentes & Não-residentes \\
\hline Argentina & 16,9 & 10,3 & 20,3 & 21,5 & 20,6 \\
\hline Brasil & 41,1 & 67,6 & 39,3 & 59,0 & 23,6 \\
\hline México & 18,7 & 8,9 & 19,6 & 7,3 & 43,6 \\
\hline Total & 76,7 & 86,9 & 79,2 & 87,8 & 87,7 \\
\hline
\end{tabular}

Fonte: Erber (2000)

Os dados apresentados por Erber ${ }^{7}$ são de 1996 e podem ser considerados desatualizados para efeito de contextualização atual da região. No entanto, seu estudo permite o delineamento de uma perspectiva histórica do desenvolvimento da América Latina e a identificação de parâmetros que servem ao entendimento do contexto mais recente. Assim, para uma análise atual da região, foram tomados por base os elementos avaliados em seu estudo e foi utilizada a mesma fonte de dados, a Ricyt. Os resultados são apresentados na Tabela 2. 
Tabela 2. A concentração de C\&T da América Latina: participação da Argentina, Brasil e México como \% do total regional -2010

\begin{tabular}{l|c|c|c|c|c}
\hline País & \multicolumn{1}{|l|}{ PIB } & $\begin{array}{l}\text { Gastos em } \\
\text { C\&T }\end{array}$ & $\begin{array}{l}\text { Publicações } \\
\text { científicas }\end{array}$ & \multicolumn{2}{l}{ Patentes concedidas } \\
\hline \multicolumn{7}{|l}{} & & Residentes & Não-residentes \\
\hline Argentina & 7,2 & 4,7 & 12,2 & 13,5 & 6,7 \\
\hline Brasil & 41,7 & 63,6 & 52,3 & 42,8 & 17,1 \\
\hline México & 20 & 7,8 & 14,7 & 14,5 & 53,3 \\
\hline Total & $\mathbf{6 8 , 9}$ & $\mathbf{7 6 , 1}$ & $\mathbf{7 9 , 2}$ & $\mathbf{7 0 , 8}$ & $\mathbf{7 7 , \mathbf { 1 }}$ \\
\hline
\end{tabular}

Fonte: Elaboração dos autores.

Ao se observar os dados referentes a $2010^{\text {iv }}$, em comparação com os dados de 1996, percebe-se que o valor relativo do PIB dos investimentos em C\&T e do número de patentes concedidas nos três países diminuiu. Em relação ao PIB, o decréscimo ocorreu em razão da diminuição do valor relativo PIB da Argentina, único país entre os três em que ele foi reduzido no período observado. Em relação aos investimentos em C\&T, observa-se uma queda de cerca de 10\% na soma dos três países analisados. Por fim, a diminuição do total da quantidade de patentes concedidas para residentes e não residentes dos três países é decorrente da queda no número relativo da Argentina e Brasil, uma vez que o México teve aumento na quantidade de patentes.

$\mathrm{O}$ valor relativo de publicações científicas ${ }^{\mathrm{v}}$ dos três países manteve-se igual nos dois períodos analisados. Esta dimensão foi observada a partir de dados do Science Citation Index (SCI), divulgados pelo Ricyt. O SCI é um índice de publicações científicas que, conforme apontaram Carpenter e Narin ${ }^{9}$, constitui uma fonte para o desenvolvimento de indicadores da atividade científica internacional. As publicações coletadas pelo índice são artigos de periódicos científicos que, segundo Perez, Mateos e De La Fuente ${ }^{10}$, representam um número reduzido e seleto das publicações científicas internacionais e seu universo restringe-se quase que exclusivamente às revistas científicas em inglês. Entretanto, ainda de acordo com os autores, apesar das limitações apontadas, o índice é utilizado por "boa parte das avaliações científicas e estudos sobre o uso de informação científica em países de todo o mundo"10 (tradução dos autores). Esta constância na quantidade de publicações científicas ocorreu devido ao aumento da quantidade de artigos brasileiros em bases de dados internacionais, uma vez que as quantidades da Argentina e do México diminuíram em porcentagem relativa.

Observa-se, assim, que a configuração latino-americana não mudou substancialmente entre 1996 e 2010, uma vez que Brasil, Argentina e México continuaram concentrando cerca de $70 \%$ dos valores do conjunto dos 24 países da região. No entanto, houve uma queda na concentração do grupo composto pelo Brasil, Argentina e México em 2010, em comparação a 1996, o que indica um acréscimo na participação dos outros países da região nas dimensões analisadas.

Outro aspecto possível de ser observado a partir dos dados disponíveis pela Ricyt é a relação do PIB com o investimento em C\&T. Em 2010, o PIB total da região era de 5.134.751,96 dólares, o que representou um aumento de 62,9\% em relação a 1996. Entretanto, o aumento não foi tão significativo em relação aos investimentos em C\&T. Em 1996, foi observado que a relação era de 0,76\%, ou seja, esta era a porcentagem do PIB usada para investimentos em C\&T. Já em 2010, a relação entre o PIB e os investimentos aumentou para 1,09\%, o que representa um aumento de 43\% em relação a 1996 (Tabela 3).

iv Os dados de 2010 são os dados completos mais atualizados disponíveis na base. http://db.ricyt.org/query/AR, $B B, B O, B R, C A, C L$,CO,CR,CU,DO,EC,ES,GT,GY,HN,HT,JM,MX,NI,PA,PE,PR,PT,PY,SV,TT,US,UY,VE,AL,IB/1990\%2C2014/PBIUSD

v Somente artigos de periódicos científicos são tratados como publicações científicas pela base SCI. 
Tabela 3. Valor do PIB investido em C\&T

\begin{tabular}{l|l|l}
\hline \multicolumn{1}{c|}{ ano } & \multicolumn{1}{|c|}{ PIB } & \multicolumn{1}{|c}{ Valor do PIB investido em C\&T } \\
\hline 1996 & $\$ 3.229 .758,00$ & $\$ 26.801,00$ \\
\hline 2010 & $\$ 5.134 .751,00$ & $\$ 55.968,00$ \\
\hline Crescimento & $\mathbf{6 2 , 9 0 \%}$ & $\mathbf{4 3 \%}$ \\
\hline
\end{tabular}

Fonte: Elaboração dos autores.

Considerando o crescimento do PIB de outros países da região, Albornoz, Macedo e Alfaraz ${ }^{11}$ incluem a Venezuela e a Colômbia no grupo dos países que concentram a maior parte do PIB da região. Assim, em 2010, a soma do PIB dos dois países, mais o do Brasil, México e Argentina, correspondia a 80\% do PIB de toda a América Latina. Para os autores, "esta concentração destaca a necessidade de estratégias de desenvolvimento muito diversas, o que, por sua vez, tem um impacto sobre o tipo de tecnologia e inovação adotada em cada país”. Entre as ações estratégicas sugeridas pelos autores destacam-se a adaptação das universidades para pensar C\&T para a sociedade, a criação de agências de fomento à pesquisa, a promoção da inovação, o estreitamento das relações entre os centros de pesquisas e os setores produtivos e a disseminação do conhecimento produzido. As estratégias de maior interesse para este estudo são as que se referem à disseminação do conhecimento científico. Segundo os mesmos autores, nas últimas décadas, a América Latina tem se mostrado afeita à tendência de democratização do conhecimento, principalmente a partir da perspectiva pública da ciência.

\section{Publicações científicas na América Latina}

Fruto do interesse público ou de outros tipos de iniciativas, as publicações científicas da América Latina em bases de dados internacionais cresceram consideravelmente na última década. De acordo com dados disponíveis na Ricyt, a quantidade de publicações cresceu mais de 100\%, entre 2000 e 2010, como mostra a Tabela 4.

Tabela 4. Crescimento da quantidade de publicações científicas da América Latina em bases de dados internacionais no período de 2000 a 2010

\begin{tabular}{l|l|l|l}
\hline Base de dados & $\mathbf{2 0 0 0}$ & $\mathbf{2 0 1 0}$ & $\begin{array}{l}\text { Crescimento } \\
\text { percentual }\end{array}$ \\
\hline SCI & 29780 & 69060 & $132 \%$ \\
\hline Pascal & 14387 & 21444 & $49 \%$ \\
\hline Inspec & 14010 & 18362 & $31 \%$ \\
\hline Compendex & 4692 & 18606 & $296 \%$ \\
\hline MEDLINE & 8609 & 23831 & $176 \%$ \\
\hline Biosis & 16412 & 27276 & $66 \%$ \\
\hline Chemical Abstracts & 13658 & 24302 & $78 \%$ \\
\hline CAB International & 10500 & 25340 & $141 \%$ \\
\hline Total & $\mathbf{1 1 2 0 4 8}$ & $\mathbf{2 2 8 2 2 1}$ & $\mathbf{1 0 4 \%}$ \\
\hline
\end{tabular}

Fonte: Elaboração dos autores 
O crescimento das publicações latino-americanas se deve, em grande parte, ao crescimento das publicações científicas no Brasil. Este dado pode ser observado a partir da análise de duas bases da Tabela 4, uma que possui a maior quantidade de publicações no ano de 2010, o Science Citation Index (SCI), e outra que apresenta a maior taxa de crescimento no período, o Compendex. Ambas indicam que cerca de metade das publicações indexadas da América Latina são de origem brasileira (52\% na SCI e 51\% na Compendex). Os dados demonstram, portanto, grande participação do Brasil na atividade científica da região que é disseminada internacionalmente (Quadro 1).

Tablea 5. Comparação da quantidade de publicações por países da América Latina das bases SCle Compendex em 2010

\begin{tabular}{|c|c|c|c|}
\hline \multicolumn{2}{|c|}{ SCI em 2010} & \multicolumn{2}{|c|}{ Compendex em 2010} \\
\hline Países & No de publicações & Países & No de publicações \\
\hline Argentina & 8469 & Argentina & 1942 \\
\hline Barbados & 69 & Barbados & 5 \\
\hline Bolívia & 220 & Bolívia & 18 \\
\hline Brasil & 36155 & Brasil & 9517 \\
\hline Chile & 5162 & Chile & 1069 \\
\hline Colômbia & 2798 & Colômbia & 832 \\
\hline Costa Rica & 456 & Costa Rica & 39 \\
\hline Cuba & 818 & Cuba & 175 \\
\hline Dominicana & 70 & Dominicana & 2 \\
\hline Equador & 350 & Equador & 69 \\
\hline Guatemala & 133 & Guatemala & 6 \\
\hline Guiana & 28 & Guayana & 1 \\
\hline Honduras & 57 & Honduras & 3 \\
\hline Haiti & 38 & Haiti & 0 \\
\hline Jamaica & 362 & Jamaica & 30 \\
\hline México & 10171 & México & 4127 \\
\hline Nicarágua & 87 & Nicarágua & 3 \\
\hline Panamá & 424 & Panamá & 40 \\
\hline Peru & 766 & Peru & 87 \\
\hline Paraguai & 78 & Paraguai & 8 \\
\hline El Salvador & 59 & El Salvador & 29 \\
\hline Trinidad y Tobago & 185 & Trinidad y Tobago & 69 \\
\hline Uruguai & 720 & Uruguai & 165 \\
\hline Venezuela & 1385 & Venezuela & 370 \\
\hline TOTAL & 69060 & TOTAL & 18606 \\
\hline
\end{tabular}

Fonte: Elaboração dos autores 
Um dos elementos que se destacam para explicar o aumento das publicações científicas brasileiras é a larga utilização do Sistema Eletrônico de Editoração de Revistas (SEER) no Brasil. Este sistema é um software para o gerenciamento de periódicos eletrônicos de acesso aberto. O SEER é a tradução brasileira do Open Jornal System (OJS) feita pelo Instituto Brasileiro de Informação em Ciência e Tecnologia (Ibict) que, segundo Moraes e Miranda' ${ }^{12}$, tem por função "agilizar o processo, reduzir custos e divulgar mundialmente as publicações científicas”. O software, que foi lançado em 2004, já favoreceu a criação de mais de mil periódicos no Brasil em meio eletrônico segundo os dados apresentados na página web ${ }^{\text {vi }}$ do sistema.

De acordo com dados do DOAJvii, o uso do SEER viii no país parece também ter contribuído para o posicionamento do Brasil em segundo lugar entre os países com maior número de periódicos de acesso aberto no mundo. Segundo dados estatísticos desse diretório, desde 2003 foram criados cerca de 80 periódicos por ano no Brasil, totalizando, no início de $2013^{\mathrm{ix}}, 807$ periódicos científicos de acesso aberto. Esta quantidade só é menor do que a apresentada pelos EUA, que na época possuía cerca de 1.270 (Tabela 6).

Tabela 6. Quantidade de periódicos no DOAJ por país

\begin{tabular}{|c|c|c|c|c|c|c|c|c|c|c|c|c|c|}
\hline & \multirow{2}{*}{ País } & \multicolumn{12}{|c|}{ Número de periódicos adicionados no DOAJ } \\
\hline & & 2002 & 2003 & 2004 & 2005 & 2006 & 2007 & 2008 & 2009 & 2010 & 2011 & 2012 & 2013 \\
\hline 1 & $\begin{array}{l}\text { Estados } \\
\text { Unidos }\end{array}$ & $\underline{16}$ & $\underline{213}$ & $\underline{298}$ & $\underline{384}$ & $\underline{435}$ & $\underline{522}$ & $\underline{699}$ & $\underline{804}$ & $\underline{962}$ & $\underline{1179}$ & $\underline{1269}$ & $\underline{1274}$ \\
\hline 2 & Brasil & 0 & $\underline{8}$ & $\underline{125}$ & $\underline{172}$ & $\underline{223}$ & $\underline{278}$ & $\underline{350}$ & $\underline{394}$ & $\underline{529}$ & $\underline{660}$ & $\underline{801}$ & $\underline{807}$ \\
\hline 3 & Reino Unido & $\underline{5}$ & $\underline{111}$ & $\underline{152}$ & $\underline{191}$ & $\underline{230}$ & $\underline{261}$ & $\underline{292}$ & $\underline{348}$ & $\underline{465}$ & $\underline{510}$ & $\underline{575}$ & $\underline{575}$ \\
\hline 4 & Índia & 0 & $\underline{14}$ & $\underline{31}$ & $\underline{45}$ & $\underline{61}$ & $\underline{78}$ & $\underline{100}$ & $\underline{148}$ & $\underline{275}$ & $\underline{368}$ & $\underline{463}$ & $\underline{472}$ \\
\hline 5 & Espanha & 0 & $\underline{5}$ & $\underline{21}$ & $\underline{79}$ & $\underline{128}$ & $\underline{155}$ & $\underline{217}$ & $\underline{244}$ & $\underline{321}$ & $\underline{393}$ & $\underline{442}$ & $\underline{446}$ \\
\hline 6 & Egito & $\underline{3}$ & $\underline{3}$ & $\underline{4}$ & $\underline{8}$ & $\underline{16}$ & $\underline{33}$ & $\underline{61}$ & $\underline{127}$ & $\underline{158}$ & $\underline{284}$ & $\underline{350}$ & $\underline{351}$ \\
\hline 7 & Alemanha & 4 & $\underline{16}$ & $\underline{36}$ & $\underline{70}$ & $\underline{96}$ & $\underline{128}$ & $\underline{155}$ & $\underline{178}$ & $\underline{214}$ & $\underline{243}$ & $\underline{260}$ & $\underline{280}$ \\
\hline 8 & Canadá & 0 & $\underline{24}$ & $\underline{35}$ & $\underline{45}$ & $\underline{60}$ & $\underline{75}$ & $\underline{101}$ & $\underline{131}$ & $\underline{177}$ & $\underline{221}$ & $\underline{255}$ & $\underline{257}$ \\
\hline 9 & Romênia & 0 & $\underline{4}$ & $\underline{5}$ & $\underline{5}$ & $\underline{12}$ & $\underline{17}$ & $\underline{29}$ & $\underline{65}$ & $\underline{145}$ & $\underline{216}$ & $\underline{249}$ & $\underline{253}$ \\
\hline 10 & Itália & 0 & $\underline{4}$ & $\underline{13}$ & $\underline{31}$ & $\underline{47}$ & $\underline{59}$ & $\underline{71}$ & $\underline{99}$ & $\underline{144}$ & $\underline{192}$ & $\underline{229}$ & $\underline{234}$ \\
\hline 11 & Turquia & 0 & $\underline{4}$ & $\underline{11}$ & $\underline{32}$ & $\underline{42}$ & $\underline{53}$ & $\underline{76}$ & $\underline{100}$ & $\underline{136}$ & $\underline{180}$ & $\underline{210}$ & $\underline{212}$ \\
\hline 12 & Colômbia & 0 & $\underline{2}$ & $\underline{4}$ & $\underline{9}$ & $\underline{28}$ & $\underline{46}$ & $\underline{64}$ & $\underline{90}$ & $\underline{109}$ & $\underline{142}$ & $\underline{201}$ & $\underline{208}$ \\
\hline 13 & França & 0 & $\underline{10}$ & $\underline{16}$ & $\underline{37}$ & $\underline{46}$ & $\underline{56}$ & $\underline{73}$ & $\underline{82}$ & $\underline{118}$ & $\underline{136}$ & $\underline{175}$ & $\underline{175}$ \\
\hline 14 & Irã & 0 & 0 & 0 & $\underline{5}$ & $\underline{11}$ & $\underline{23}$ & $\underline{34}$ & $\underline{44}$ & $\underline{75}$ & $\underline{120}$ & $\underline{167}$ & $\underline{171}$ \\
\hline 15 & Polônia & 0 & $\underline{9}$ & $\underline{13}$ & $\underline{21}$ & $\underline{30}$ & $\underline{37}$ & $\underline{56}$ & $\underline{62}$ & $\underline{78}$ & $\underline{126}$ & $\underline{142}$ & $\underline{144}$ \\
\hline 16 & Chile & 0 & $\underline{3}$ & $\underline{44}$ & $\underline{63}$ & $\underline{76}$ & $\underline{83}$ & $\underline{97}$ & $\underline{107}$ & $\underline{121}$ & $\underline{127}$ & $\underline{141}$ & $\underline{142}$ \\
\hline 17 & Argentina & 0 & 0 & $\underline{1}$ & $\underline{9}$ & $\underline{20}$ & $\underline{34}$ & $\underline{48}$ & $\underline{56}$ & $\underline{84}$ & $\underline{110}$ & $\underline{134}$ & $\underline{136}$ \\
\hline 18 & Suíça & $\underline{1}$ & $\underline{8}$ & $\underline{12}$ & $\underline{20}$ & $\underline{23}$ & $\underline{24}$ & $\underline{42}$ & $\underline{56}$ & $\underline{79}$ & $\underline{103}$ & $\underline{134}$ & $\underline{134}$ \\
\hline 19 & México & 0 & $\underline{1}$ & $\underline{5}$ & $\underline{28}$ & $\underline{44}$ & $\underline{56}$ & $\underline{70}$ & $\underline{78}$ & $\underline{88}$ & $\underline{106}$ & $\underline{126}$ & $\underline{126}$ \\
\hline 20 & Austrália & 0 & 15 & 28 & 39 & 46 & 56 & 64 & 77 & 97 & 118 & 123 & 124 \\
\hline
\end{tabular}

Fonte: Elaboração dos autores

vi SEER. Disponível em: http://seer.ibict.br/index.php?option=com mtree\&ltemid=109.

vii O DOAJ é um diretório internacional construído de forma colaborativa para a disponibilização de informações de periódicos acadêmicos com revisão pelos pares e em acesso aberto. Disponível em: https://doaj.org/.

viii O SEER é uma versão do software OJS, destinado à construção e ao gerenciamento de periódicos acadêmicos. A versão SEER foi traduzida para o idioma português e é distribuída para os editores brasileiros por meio de ações do Instituto Brasileiro de Informação em Ciência e Tecnologia (Ibict). Disponível em: http://www.ibict.br/seer.

ix Dados de janeiro de 2013. Disponível em: https://doaj.org/oainfo 
Após o Brasil, o próximo país da América Latina a aparecer nessa lista é a Colômbia, com 208 periódicos, seguida do Chile, com 142, da Argentina, com 136, e do México, com 126. A quantidade total de periódicos registrados nesse diretório, cuja origem são países latino-americanos, é de 1.546 (Tabela 7). Devido à ausência de informações no diretório DOAJ, a soma não incluiu dados de seis países da região considerados por este estudo: Guiana, Honduras, Haiti, Panamá, El Salvador e Trinidad y Tobago.

Tabela 7. Quantidade de periódicos latino-americanos no DOAJ

\begin{tabular}{|c|c|c|c|c|c|c|c|c|c|c|c|}
\hline & País & 2003 & 2004 & 2005 & 2006 & 2007 & 2008 & 2009 & 2010 & 2011 & 2012 \\
\hline 2 & Brasil & 8 & 119 & 166 & 217 & 273 & 346 & 390 & 525 & 656 & 768 \\
\hline 12 & Colômbia & 2 & 4 & 9 & 28 & 46 & 64 & 86 & 107 & 140 & 189 \\
\hline 16 & Chile & 3 & 44 & 63 & 76 & 83 & 14 & 97 & 111 & 117 & 130 \\
\hline 17 & Argentina & 0 & 1 & 9 & 20 & 34 & 48 & 56 & 84 & 110 & 132 \\
\hline 18 & México & 1 & 5 & 27 & 43 & 52 & 64 & 72 & 82 & 100 & 120 \\
\hline 25 & Venezuela & 3 & 38 & 43 & 58 & 60 & 66 & 72 & 77 & 78 & 85 \\
\hline 34 & Cuba & 0 & 13 & 17 & 19 & 20 & 21 & 23 & 30 & 41 & 48 \\
\hline 48 & Peru & 0 & 0 & 3 & 8 & 10 & 14 & 18 & 20 & 22 & 26 \\
\hline 51 & Costa Rica & 0 & 4 & 4 & 6 & 6 & 11 & 12 & 15 & 22 & 25 \\
\hline 70 & Bolívia & 0 & 0 & 0 & 0 & 0 & 1 & 1 & 2 & 3 & 7 \\
\hline 73 & Uruguai & 0 & 0 & 0 & 0 & 0 & 1 & 3 & 4 & 4 & 5 \\
\hline 83 & Guatemala & 0 & 0 & 0 & 2 & 2 & 2 & 2 & 2 & 1 & 3 \\
\hline 94 & Nicarágua & 0 & 0 & 0 & 0 & 0 & 1 & 1 & 1 & 1 & 2 \\
\hline 96 & Paraguai & 0 & 0 & 0 & 0 & 0 & 0 & 1 & 1 & 1 & 2 \\
\hline 99 & Jamaica & 0 & 0 & 0 & 0 & 0 & 0 & 1 & 1 & 1 & 1 \\
\hline 102 & $\begin{array}{l}\text { República } \\
\text { Dominicana }\end{array}$ & 0 & 0 & 0 & 0 & 0 & 1 & 1 & 1 & 1 & 1 \\
\hline 105 & Equador & 0 & 0 & 0 & 0 & 1 & 1 & 1 & 1 & 1 & 1 \\
\hline 110 & Barbados & 0 & 0 & 1 & 1 & 1 & 1 & 1 & 1 & 1 & 1 \\
\hline \multicolumn{11}{|c|}{ Total } & 1.546 \\
\hline
\end{tabular}

Fonte: Elaboração dos autores

O total de 1.546 revistas científicas latino-americanas, a princípio, parece ser bastante expressivo para considerar o desenvolvimento de ações para consolidação da estratégia de acesso aberto denominada via dourada (acesso aberto por meio da publicação de periódicos científicos de acesso aberto). No entanto, outras questões referentes à qualidade, ao impacto e à visibilidade dessas publicações devem ser levadas em consideração para uma análise mais profunda em estudos específicos sobre o fenômeno. 


\section{Financiamento da ciência na América Latina}

Além do amplo uso de software para a criação de periódicos científicos de acesso aberto, outro fator que pode ter influência na participação da região nas ações de democratização do conhecimento científico é a própria origem dos recursos de financiamento da ciência que se produz na América Latina. Um dos argumentos utilizados pelo Movimento de Acesso Aberto para a defesa da disponibilização livre da literatura científica é que boa parte dela é resultante de financiamento público. Esta característica parece ser mais acentuada na América Latina, onde, segundo dados da Unesco ${ }^{13}$, cerca de dois terços dos investimentos em pesquisa são advindos de recursos públicos.

De acordo com os dados disponíveis no Ricyt, em 2010, cerca de metade dos recursos de investimento em pesquisa eram de origem direta do Estado, a outra metade tinha origem em empresas públicas e privadas (Tabela 8).

Tabela 8. Financiamento de atividades científicas e tecnológicas na América Latina

\begin{tabular}{l|l}
\hline Financiamento por setor & $\mathbf{2 0 1 0}$ \\
\hline Governo & $48,64 \%$ \\
\hline Empresas (Públicas e Privadas) & $47,98 \%$ \\
\hline Educação Superior & $2,51 \%$ \\
\hline Organização privada sem fins lucrativos & $0,39 \%$ \\
\hline Exterior & $0,49 \%$ \\
\hline
\end{tabular}

Fonte: Elaboração dos autores

Apesar dos dados apresentados pela Ricyt indicarem que cerca de 47\% dos investimentos em C\&T são provenientes de empresas públicas e privadas, uma análise preliminar das instituições citadas pela própria Ricyt demonstra que as principais instituições de promoção das atividades de ciência, tecnologia e inovação (CT\&I) são dos setores governo, empresa pública ou empresa pública e privada. Dessa forma, nenhuma das organizações citadas se enquadra exclusivamente no setor empresa privada, como demonstrado no Quadro 1. Sobre os dados referentes ao setor, é importante destacar que eles foram levantados de forma preliminar, a partir da análise da descrição de cada instituição feita na página da Ricyt ou na página web das próprias instituições.

Quadro 2. Organismos de promoção das atividades de CT\& dos países da América Latina

\begin{tabular}{|c|c|c|}
\hline País & Organismo de promoção das atividades de C,T\&I & Setor \\
\hline \multirow{2}{*}{ Argentina } & Consejo Nacional de Investigaciones Científicas y Técnicas (CONICET) & Governo \\
\hline & Agencia Nacional de Promoción Científica y Tecnológica (ANPCYT) & Governo \\
\hline Bolívia & Não existem organismos relevantes nesta categoria & -- \\
\hline \multirow{6}{*}{ Brasil } & Conselho Nacional de Desenvolvimento Científico e Tecnológico (CNPq) & Governo \\
\hline & Financiadora de Estudos e Projetos (Finep) & Empresa pública \\
\hline & Coordenação de Aperfeiçoamento de Pessoal de Nível Superior (Capes) & Governo \\
\hline & Banco Nacional de Desenvolvimento Econômico e Social (BNDES) & Empresa pública \\
\hline & Agencia Brasileira de Desenvolvimento Industrial (ABDI) & Governo \\
\hline & Fundações estaduais de apoio a ciência, tecnologia e inovação & Governo \\
\hline
\end{tabular}


Quadro 2. Organismos de promoção das atividades de CT\& dos países da América Latina (continuação)

\begin{tabular}{|c|c|c|}
\hline País & Organismo de promoção das atividades de C,T\&I & Setor \\
\hline \multirow{4}{*}{ Chile } & Comisión Nacional de Investigación Científica y Tecnológica (CONICYT) & Empresa pública \\
\hline & Corporación de Fomento de la Producción (CORFO) & Governo \\
\hline & Iniciativa Científica Milenio (ICM) & Governo \\
\hline & Fundación para la Innovación Agraria (FIA) & Governo \\
\hline \multirow{2}{*}{ Colômbia } & $\begin{array}{l}\text { Departamento Administrativo de Ciencias, Tecnología e Innovación - } \\
\text { COLCIENCIAS }\end{array}$ & Governo \\
\hline & $\begin{array}{l}\text { Fondo Nacional de Financiamiento para la Ciencia, la Tecnología y la } \\
\text { Innovación, Fondo Francisco José de Caldas }\end{array}$ & Empresa pública \\
\hline \multirow{2}{*}{ Costa Rica } & Consejo Nacional de Investigaciones Científicas y Tecnológicas (CONICIT) & Governo \\
\hline & Comisión de Incentivos & Governo \\
\hline Equador & Fundación para la Ciencia y Tecnología (FUNDA-CYT) & Empresa Pública Privada \\
\hline El Salvador & Não existem organismos relevantes nesta categoria. & -- \\
\hline \multirow{4}{*}{ Guatemala } & Fondo Nacional de Ciencia y Tecnología (FONACYT) & Governo \\
\hline & Programa de Apoyo a la Innovación Tecnológica (PROINTEC) & Governo \\
\hline & Fondo Competitivo de Desarrollo Tecnológico Agroalimentario (AGROCYT) & Governo \\
\hline & Comisiones Técnicas Sectoriales e Intersectoriales & Governo \\
\hline \multirow{3}{*}{ Honduras } & Consejo Hondureño de Ciencia y Tecnología (COHCIT) & Governo \\
\hline & Comisión Nacional de Competitividad & Empresa pública e privada \\
\hline & Scientific Research Council (SRC) & Governo \\
\hline \multirow{2}{*}{ México } & Consejo Nacional de Ciencia y Tecnología (CONACYT) & Governo \\
\hline & Consejos y organismos estatales de ciencia y tecnología & Governo \\
\hline Nicarágua & Consejo Nicaragüense de Ciencia y Tecnología (CONICYT) & Governo \\
\hline Panamá & $\begin{array}{l}\text { Fondo Nacional para el Desarrollo de la Ciencia, la Tecnología y la } \\
\text { Innovación (FONACITI) }\end{array}$ & Governo \\
\hline Paraguai & Fondo Nacional de Ciencia y Tecnología (FONACYT) & Governo \\
\hline \multirow{3}{*}{ Peru } & $\begin{array}{l}\text { Fondo para la Innovación, Ciencia y Tecnología (FINCYT) (Presidencia del } \\
\text { Consejo de Ministros) }\end{array}$ & Empresa Pública Privada \\
\hline & $\begin{array}{l}\text { Innovación y Competitividad para el Agro Peruano (INCAGRO) (Ministerio } \\
\text { de Agricultura) }\end{array}$ & Governo \\
\hline & $\begin{array}{l}\text { Fondo Nacional de Desarrollo Científico, Tecnológico y de Innovación } \\
\text { Tecnológica }\end{array}$ & Governo \\
\hline $\begin{array}{l}\text { República } \\
\text { Dominicana }\end{array}$ & $\begin{array}{l}\text { Fondo Nacional de Innovación y Desarrollo Científico y Tecnológico } \\
\text { (FONDOCYT) }\end{array}$ & Governo \\
\hline \multirow{2}{*}{$\begin{array}{l}\text { Trinidad y } \\
\text { Tobago }\end{array}$} & Ministry of Science Technology and Tertiary Education & Governo \\
\hline & $\begin{array}{l}\text { National Institute of Higher Education, Research, Science and Technology } \\
\text { (NIHERST) }\end{array}$ & Governo \\
\hline Uruguai & Agencia Nacional de Investigación e Innovación (ANII) & Empresa pública \\
\hline \multirow{2}{*}{ Venezuela } & Fondo Nacional de Ciencia, Tecnología e Innovación (FONACIT) & Governo \\
\hline & Fundaciones para el Desarrollo de la Ciencia y la Tecnología (FUNDACITEs) & Governo \\
\hline
\end{tabular}

Fonte: Elaboração dos autores 
Os dados apresentados no Quadro 1 corroboram o argumento sobre a natureza pública do financiamento da pesquisa na América Latina. Para Babini ${ }^{14}$, essa característica possibilitou passos importantes no sentido de promover o acesso aberto. A autora apontou o projeto de lei brasileiro e a lei Argentina como bons exemplos de como a discussão tem avançado na esfera pública na região. Somado a estes, destaca-se ainda a lei peruana de acesso aberto aprovada em 2013.

No caso do projeto de lei brasileiro, Babini ${ }^{14}$ se refere ao PL 1.120 de 2007, que propõe o estabelecimento de uma lei nacional em prol da obrigatoriedade da construção de repositórios digitais por parte das instituições de ensino superior e unidades de pesquisa de caráter público. O objetivo do PL era a construção de repositórios digitais e a obrigatoriedade de depósito de uma cópia dos trabalhos produzidos por pesquisadores que recebessem financiamento público. O PL teve todos os pareceres favoráveis à sua aprovação, com algumas emendas, mas em 2011 foi arquivado porque tramitou por quatro anos no Congresso e não teve sua aprovação até o final de uma legislatura ${ }^{x}$. No mesmo ano, outro projeto de lei (PLS 387/2011) foi apresentado em substituição ao primeiro, agora pelo Senado Federal. O seu conteúdo principal é idêntico ao do PL de 2007, no entanto traz algumas especificações a mais, como o tipo de documento que deve ser depositado e o tempo entre a publicação e o depósito. O novo PL ainda não foi aprovado, mas, segundo consta no Portal Atividade Legislativa do Senado Federal ${ }^{x i}$, o PL está em tramitação em diversas pautas das comissões da Casa.

A lei argentina refere-se ao Sistema Nacional de Repositórios Digitais (SNRD) criado em 2011 por meio de uma resolução ministerial. O seu propósito é criar uma rede interoperável de repositórios digitais, por meio do estabelecimento de políticas, padrões e protocolos comuns. No mesmo ano, foi elaborado um projeto de lei para garantir o desenvolvimento de repositórios digitais em instituições que recebem financiamento público, bem como o depósito de cópias dos trabalhos revisados por pares, publicados ou não. Segundo a notícia publicada no website do Ministerio de Ciencia, Tecnología e Innovación Productiva ${ }^{\text {xii }}$ argentino, a lei foi sancionada em maio de 2012. A novidade desta lei em relação ao PL brasileiro é a especificação para o tratamento dos dados primários de pesquisa.

A lei peruana de promoção do acesso aberto (Lei 30035/2013) foi aprovada em 2013 e regula o Repositorio Nacional Digital de Ciencia, Tecnología y Innovación de Acceso Abierto. O repositório em questão é definido como um 'sítio centralizado', onde se mantém informação digital resultante da produção de ciência, tecnologia e inovação no país. O seu uso é destinado a pesquisadores vinculados a órgãos do Estado, pesquisadores que receberam financiamento público e pesquisadores que desejam disponibilizar sua produção em acesso aberto.

Leis nacionais como essas certamente são poderosas aliadas para garantia da criação e/ou a consolidação de mecanismos que promovam o acesso aberto. Portanto, Alperin, Fishman e Willinsky ${ }^{15}$ consideram que a América Latina já tem desenvolvido consideravelmente suas ações para a promoção do acesso aberto e que isso pode ser favorável para a superação dos limites da região no que se refere à visibilidade de sua produção científica. Sobre a questão, os autores afirmam que "muitos avanços têm sido feitos na América Latina para promover estes canais, talvez mais do que em qualquer outra região do mundo".

\section{Conclusões}

O modelo de colonização europeia, particularmente da Espanha e Portugal, na América Latina influenciou consideravelmente o ritmo e o modo de desenvolvimento das atividades de C\&T. Avanços

\footnotetext{
x O arquivamento foi baseado no artigo 105 do Regimento Interno da Câmara do Deputados. Disponível em: http://kuramoto. blog.br/2011/03/10/pl-11202007-foi-arquivado-indevidamente/.

xi Disponível em: http://www.senado.gov.br/atividade/materia/detalhes.asp?p cod mate=101006.

xii Disponível em: http://www.mincyt.gov.ar/noticias/noticias detalles.php?id noticia=959.
} 
científicos e tecnológicos de povos originais em diversos campos foram extintos, momento em que se instaurou a dinâmica de importação tanto de conhecimentos quanto de tecnologias. Uma vez que a origem do conhecimento científico relevante passou a ser a Europa, e posteriormente suas colônias de desenvolvimento, os canais de comunicação de maior valor, consequentemente, são originários dos grandes centros produtores, marcando profundamente o sistema de comunicação científica da região.

Em razão de sua desestruturação histórica associada a outros diversos motivos, o sistema de comunicação científica da região não foi acompanhado pelo progresso de uma indústria, a exemplo da Europa e EUA. Se por um lado durante muito tempo esse fato contribuiu para a invisibilidade da ciência produzida na região, por outro, mais recentemente, foi exatamente o fator que contribuiu para a bem-sucedida estratégia de ampla comunicação científica por meio de periódicos de acesso aberto. É evidente, no entanto, que os avanços nesse campo devem também ser avaliados em razão da qualidade dos periódicos.

É possível concluir que há um evidente protagonismo brasileiro no que se refere aos avanços em C\&T, e sua comunicação, na região. Os indicadores demonstram que o país responde por quantidade significativa dos resultados de pesquisa disseminados internacionalmente.

Também é importante destacar, conforme indicado pelo cenário descrito, que a maior parte da atividade científica e tecnológica da América Latina é financiada com recursos públicos. Tal característica contribui para imprimir na região a vocação para uma comunicação científica aberta, cumpridora de suas funções básicas, tal como argumentado por Roosendaal e Geurts ${ }^{1}$, Kaplan e Storer ${ }^{16}$ e Menzel ${ }^{17-18}$.

Por fim, considera-se que o acesso aberto representa um elemento importante para a disseminação internacionalizada da ciência produzida na América Latina, além da contribuição na promoção da cooperação Sul/Sul e Sul/Norte, até então obstaculizados. Como demonstrado pelo cenário descrito, a região tem apresentado consideráveis avanços nos últimos dez anos, tanto em investimento em pesquisa como no desenvolvimento de mecanismos para a comunicação científica.

\section{Referências}

1. Roosendaal HE, Geurts PATM. Forces and functions in scientific communication: an analysis of their interplay [Internet]. In: Conference on Co-Operative Research in Information Systems in Physics; 1997 Sept. 1-3; Oldenburg: University of Oldenburg; 2017 [cited 2016 June 22]. Available from: http://doc. utwente.nl/60395/.

2. Garvey WD. Communication: the essence of science: facilitating information exchange among librarians, scientists, engineers and students. [place unknown]: Elsevier; 2014.

3. Chan L, Costa S. Participation in the global knowledge commons: challenges and opportunities for research dissemination in developing countries. New Libr World [Internet]. 2005 [cited 2013 Feb. 06];106(3-4)141-63. Available from: 10.1108/03074800510587354

4. Fernandes LA, Gomes JMM. Relatórios de pesquisa nas ciências sociais: características e modalidades de investigação. ConTexto. 2003;3(4):1-23.

5. Guimaraes E. Política de línguas na América Latina. In: Políticas linguísticas para América Latina: actas del congreso internacional; 1997 nov. 26-29; Buenos Aires: Facultad de Filosofía y Letras; 1991.

6. Lopes J. Que ciência para América Latina na era da globalização?. Interciência. 1999;24(3): 190-1.

7. Erber F. Perspectivas da América Latina em ciência e tecnologia. Parc Estratég. 2000;5(8)181-0.

8. Zuñiga A. Historia de la ciência y la tecnologia y la realidad de América Latina. Elementos. 1986;6(1)17-2.

9. Carpenter MP, Narin F. The adequacy of the science citation index (SCI) as an indicator of international scientific activity. J Am Soc Inform Sci. 1981 Nov.;32(6):430-9.

10. Hernández Pérez T, Rodríguez Mateos, D; Bueno de la Fuente G. Open access: el papel de las bibliotecas en los repositorios institucionales de acceso abierto. Anales de Documentación. 2007; 10;185-204. 
11. Albornoz M, Macedo M, Alfaraz C. Latin America. In: Organización de Las Naciones Unidas para la Educación, la Cidadania y la Cultura. Informe de la Unesco sobre la ciência 2010: el estado actual de la ciência en el mundo. França; 2010.

12. Moraes M, Miranda A. Produção do conhecimento sobre o Sistema Eletrônico de Editoração de Revistas (SEER) no Brasil nos anos de 2003 a 2010. Encontros Bibli: R Eletron de Bibliot e Ci da Inf [Internet]. 2011 [citado em 2013 fev. 02];16(32):27-40. Disponível em: DOI:10.5007/15182924.2011v16n32p27.

13. UNESCO. Informe de la Unesco sobre la ciência 2010: el estado actual de la ciência en el mundo [internet]. Unesco; 2010. [citado 01 fev 2013]. Disponível em: <http://www.unesco.org/new/fileadmin/ MULTIMEDIA/HQ/SC/pdf/sc_usr10_la_EN.pdf>.

14. Babini D. Acceso abierto y visibilidad de publicaciones científicas en América Latina [Internet]. In: Proceedings of 11th Jornadas de Investigación; 2012 enero 12-13 [cited 2013 Feb. 02]. Temuco, Chile: University Catolic de Temuco; 2012. Disponível em: http://eprints.rclis.org/17903/.

15. Alperin JP, Fischman G, Willinsky, J. Acesso livre e publicação acadêmica na América Latina: dez sabores e algumas reflexões. Liinc em R [Internet]. 2008 set. [citado em 2016 dez. 12];4(2)172-85. Disponível em: http://revista.ibict.br/liinc/article/view/3165/2831 .

16. Kaplan N, Storer NW. Scientific communication. In: Sills DL. International encyclopedia of the social sciences. New York: Macmillan; 1968. v.14, p.112-7.

17. Menzel $\mathrm{H}$. The flow of information among scientists: problems, opportunities and research questions. [New York] : Columbia University, Bureau of Applied Social Research, 1958.

18. Menzel H. Scientific communication: five themes from social science research. Am Psychol. $1966 ; 21(10) ; 999-04$. 